\title{
Rigidez muscular, trastorno de sensorio y antipsicóticos: Reporte de caso
}

Víctor Ramos-Cabrera 1,a; Fanny Ramírez-Calderón*1,a

\section{RESUMEN}

En los servicios de emergencias que atienden a una gran población, muchas veces se encuentran cuadros aislados, como deshidratación severa, hipernatremia o hiponatremia, injuria renal aguda, trastorno de sensorio, entre otras. Sin embargo, muchos de estos cuadros forman parte de una sola patología, y si no se hace una unidad clínica en el momento adecuado, la morbimortalidad se puede incrementar drásticamente. Sobre todo, en enfermedades con baja frecuencia, muy raras o poco habituales, como la que se narrará a continuación, podría empeorar el estado crítico del paciente si no se detecta a tiempo o no se maneja adecuadamente.

Este caso clínico es el de una paciente mujer de 74 años, con antecedente de patología psicótica con tratamiento irregular que, tras ingerir carbamazepina y risperidona, presenta los síntomas típicos y poco frecuentes del síndrome neuroléptico maligno (SNM).

Palabras clave: Síndrome neuroléptico maligno; Antipsicóticos; Rigidez muscular; Rabdomiólisis (Fuente: DeCS BIREME).

\section{Muscle rigidity, sensory disorder and antipsychotics: a case report}

\section{ABSTRACT}

In emergency rooms with large numbers of assigned population, we often see isolated conditions, such as severe dehydration, hypernatremia or hyponatremia, acute kidney injury, sensory disorder, among others. However, many of these conditions are part of a single disease and, if they are not gathered together as a medical unit at the right time, morbidity and mortality may drastically increase, especially in the event of diseases with a very rare or unusual frequency. Such is the case of the following disorder, which could worsen the critical condition of the patient if it is not detected on time or not treated appropriately. This clinical case addresses a 74-year-old female patient who reports a history of psychotic disorder with irregular treatment and, after the intake of carbamazepine and risperidone, presents the typical and infrequent symptoms of neuroleptic malignant syndrome (NMS).

Keywords: Neuroleptic malignant syndrome; Antipsychotic agents; Muscle rigidity; Rhabdomyolysis (Source: MeSH NLM).

1. Hospital Edgardo Rebagliati Martins EsSalud, Departamento de Emergencia de Adultos. Lima, Perú.

a. Médico especialista en Medicina de Emergencias y Desastres. 


\section{INTRODUCCIÓN}

El síndrome neuroléptico maligno (SNM) es una complicación nefasta que se manifiesta de manera idiosincrática frente a la administración de antipsicóticos. Es un cuadro clínico caracterizado por cambios de estado mental como bradipsiquia, rigidez muscular generalizada, fiebre y disautonomía, potencialmente mortal hasta en $11,6 \%$ de los casos, y se relaciona con agentes que alteran la neurotransmisión del sistema dopaminérgico, con una frecuencia estimada de 0,5-1 \% en los pacientes tratados con neurolépticos ${ }^{(1)}$. Los hallazgos de laboratorio consisten en elevación de los niveles plasmáticos de lesión muscular como creatinakinasa (CK), marcadores de reacción inflamatoria tipo leucocitosis, además de múltiples trastornos como injuria renal, alteración de electrolitos, medio interno e insuficiencia respiratoria ${ }^{(2)}$.

Presentamos el caso clínico de una paciente adulta que ingresa con marcada deshidratación, además de hipotensión arterial, asociada a orina patológica (leucocituria en sedimento urinario), por lo que recibe tratamiento para sepsis con foco infeccioso en vías urinarias. Sin embargo, el trastorno del sensorio, la rigidez de extremidades, la falla renal secundaria a rabdomiólisis, y los exámenes de laboratorio y el antecedente de uso de neurolépticos nos condujo a plantearnos un síndrome neuroléptico maligno como posibilidad diagnóstica. Este reporte de caso fue elaborado siguiendo la guía CARE Report ${ }^{(2)}$.

\section{CASO CLÍNICO}

Paciente mujer de 74 años de edad, natural y procedente de Lima (Perú), con antecedente de esquizofrenia paranoide, hace 10 años aproximadamente, con tratamiento y control irregular, familiar refiere que hace 2 semanas toma risperidona y carbamazepina. Dos días antes de su ingreso presenta desorientación, dificultad para la marcha, no logra flexionar los miembros inferiores y presenta un mayor deterioro del sensorio.
En la exploración física encuentran presión arterial 90/50 $\mathrm{mmHg}$, frecuencia cardiaca de 60 latidos por minuto, frecuencia respiratoria de 12 respiraciones por minuto y temperatura $36,5{ }^{\circ} \mathrm{C}$. Piel y mucosas secas $+++/+++$. Neurológico: tendencia al sopor, hiperreactiva. Escala de coma de Glasgow: Motor (5), verbal (3), ocular (2): 10/15M. Tórax y pulmones: murmullo vesicular pasa en ambos hemitórax. Cardiológico: I y II ruidos rítmicos, no soplos. Abdomen: blando depresible, no dolorosos, ruidos hidroaéreos disminuidos.

Con la anamnesis ampliada, los antecedentes y los exámenes de laboratorio solicitados se plantearon tres problemas diagnósticos. Primero, un trastorno de sensorio, asociado a la severa deshidratación con trastornos hidroelectrolíticos como hiponatremia hipotónica; una rabdomiólisis secundaria que desarrolla injuria renal aguda por pigmentos; y finalmente, una falla hepática aguda exacerbada por una posible sepsis de foco urinario o inicio de disfunción multiorgánica.

La paciente fue evaluada por el Servicio de Nefrología, que indicó terapia con fluidos y control bioquímico, pero no se consideró como tributaria de diálisis.

El tratamiento inicial en la Unidad de Áreas Críticas de Emergencia se enfocó en una patología séptica. Se empleó fluidoterapia y cobertura antibiótica. Con los resultados, se orientó a la rabdomiólisis y la injuria renal aguda, se evitó el taponamiento renal por pigmentos y la reposición de electrolitos. Se administró solución fisiológica ( $\mathrm{ClNa} 9 \%$ en $1000 \mathrm{cc})$ a 4 litros en 3 horas, poligelina 3,5 \% (500 cc); cobertura antibiótica previa a cultivos, con ceftriaxona 2 g por vía endovenosa cada 24 horas, clindamicina $600 \mathrm{mg}$ cada $8 \mathrm{~h}$ endovenosa. Además, omeprazol $40 \mathrm{mg}$ endovenoso cada 24 horas y gluconato de calcio al $10 \%$ endovenoso cada 8 horas.

Dentro de los exámenes de laboratorio se estudiaron el hemograma, los gases arteriales, los niveles bioquímicos, sedimento urinario y los parámetros de daño muscular (Tabla 1).

Tabla 1. Exámenes de laboratorio

\begin{tabular}{|ccc|}
\hline & Resultado & Valor referencial \\
\hline Gases arteriales & \\
\hline $\mathbf{p H}$ & 7,4 \\
$\mathrm{CO} 2$ & 32 \\
\hline $\mathrm{HCO}_{3} \mathrm{Na}$ & $20 \mathrm{mmHg}$ \\
\hline Lactato & $2 \mathrm{mmol} / \mathrm{l}$ \\
\hline
\end{tabular}




\begin{tabular}{|c|c|c|}
\hline & Resultado & Valor referencial \\
\hline \multicolumn{3}{|l|}{ Hemograma } \\
\hline Leucocitos & 24000 & $4000-11000$ \\
\hline Abastonados & 6 & $0,0-0,5$ \\
\hline Hemoglobina & 13 & $14-18$ \\
\hline Hematocrito & 34 & \\
\hline Plaquetas & 274 & $130-400$ \\
\hline \multicolumn{3}{|l|}{ Bioquímica- electrolitos } \\
\hline Glucosa & $120 \mathrm{mg} / \mathrm{dl}$ & $70-100$ \\
\hline Urea & $113 \mathrm{mg} / \mathrm{dl}$ & $22-75$ \\
\hline Creatinina & $3.1 \mathrm{mg} / \mathrm{dl}$ & $0,5-0,8$ \\
\hline TGO & $2574 \mathrm{U} / \mathrm{L}$ & $0,0-34$ \\
\hline TGP & $1605 \mathrm{U} / \mathrm{L}$ & $10-49$ \\
\hline Bilirrubina total & $0,62 \mathrm{md} / \mathrm{dl}$ & $0,30-1,20$ \\
\hline Bilirrubina directa & $0,20 \mathrm{mg} / \mathrm{dl}$ & \\
\hline Bilirrubina indirecta & $0,32 \mathrm{mg} / \mathrm{dl}$ & \\
\hline Sodio & $123 \mathrm{mmol} / \mathrm{L}$ & $135-145$ \\
\hline Potasio & $3,86 \mathrm{mmol} / \mathrm{L}$ & $3,50-5,50$ \\
\hline Calcio & $6,50 \mathrm{mg} / \mathrm{dl}$ & $8,30-10,20$ \\
\hline Osmolaridad & 271 mosml & $290-310$ \\
\hline \multicolumn{3}{|l|}{ Sedimento de orina } \\
\hline Leucocitos & $>100$ & \\
\hline Nitritos & Negativo & \\
\hline Esterasa leucocitaria & $\mathrm{PO} 3+$ & \\
\hline \multicolumn{3}{|l|}{ Marcadores de rabdomiólisis } \\
\hline Creatinfosfokinasa (CPK) & 47727 & $33-211$ \\
\hline Lactatodeshidrogenasa & 2268 & $120-246$ \\
\hline Troponina I & 0,089 & $0,010-0,023$ \\
\hline CPKMB & $>500$ & $2,0-7,0$ \\
\hline Mioglobina & $>900$ & $23-112$ \\
\hline
\end{tabular}

La paciente persiste con marcada rigidez de miembros superiores tipo rueda dentada, así como en miembros inferiores, además con signos de deshidratación; se amplía la anamnesis con los familiares quienes refieren que hace 2 semanas la paciente recibe tratamiento antipsicótico (risperidona, carbamazepina) en forma no controlada $y$, posterior a ello, presenta la sintomatología referida. Al realizar unidad clínica, se plantea el diagnóstico de síndrome neuroléptico maligno y se solicitan marcadores serológicos de rabdomiólisis entre ellos CPK-MB, creatinfosfokinasa (CPK), lactato deshidrogenasa (LHD), TGO (Tabla 1).
En el electrocardiograma se observó ritmo sinusal, con línea isoeléctrica, frecuencia cardiaca de 71 latidos por minuto con extrasístoles ventriculares no significativas. La radiografía de tórax muestra campos pulmonares libres sin evidencia de signos de consolidación, silueta cardiaca en línea media sin crecimiento de cavidades, ángulos costo frénicos libres. De igual modo, en la tomografía encefálica se observa parénquima cerebral homogéneo con buena diferenciación cortico subcortical, sin colapso de ventrículos, tampoco se logra diferenciar imágenes ocupantes de masa encefálica. 
Con los antecedentes médicos referidos (consumo de antipsicóticos), el examen clínico y los hallazgos de laboratorio y descartando otras patologías causales del cuadro, se llega al diagnóstico final de síndrome neuroléptico maligno, exacerbado por el foco séptico en vías urinarias. La paciente recibe el tratamiento de soporte con fluidoterapia, oxigenoterapia, nefroprotección y neuroprotección. Presenta una evolución lenta pero favorable, y es hospitalizada en el Servicio de Medicina por 16 días y dada de alta con evolución y pronóstico favorable.

\section{DISCUSIÓN}

El SNM es una complicación grave asociada al tratamiento con antipsicóticos (Tabla 2). Suele aparecer entre las 24-72 horas tras el inicio o cambio en el tratamiento $y$, habitualmente, se presenta con fármacos que bloquean receptores dopaminérgicos ${ }^{(3)}$. Si bien es cierto que la incidencia de este cuadro es baja, tiene un valor estimado de 0,5-1 \% de los pacientes tratados con neurolépticos. Las cuatro manifestaciones clínicas principales son hipertermia, rigidez muscular severa, disfunción del sistema nervioso autónomo y alteración del nivel de conciencia ${ }^{(4)}$. Se debe tener presente, en el diagnóstico diferencial, principalmente la patología infecciosa que, incluso, podría desencadenar el síndrome neuroléptico maligno. Además, cabe precisar que el SNM abarca múltiples problemas como el estado severo de deshidratación, alteración hidroelectrolítica que conlleva a la injuria renal aguda ${ }^{(3,5)}$, y el caso de nuestro estudio no es la excepción.

Tabla 2. Medicamentos asociados con síndrome neuroléptico maligno

\begin{tabular}{lllll}
$\begin{array}{c}\text { Neurolépticos } \\
\text { típicos }\end{array}$ & $\begin{array}{c}\text { Neurolépticos } \\
\text { atípicos }\end{array}$ & Antieméticos & $\begin{array}{c}\text { Agentes } \\
\text { dopaminérgicos } \\
\text { (abstinencia) }\end{array}$ & Otros \\
\hline $\begin{array}{l}\text { Haloperidol } \\
\text { Clorpromazina }\end{array}$ & Olanzapina & Droperidol & Levodopa & Tetrabenazina \\
\hline $\begin{array}{l}\text { Fluphenazine } \\
\text { Tioridazina }\end{array}$ & Risperidona & Metoclopramida & Amantadina & Reserpine \\
\hline Trifluordazine & Ziprasidone & Agonistas de & Amoxapine \\
\hline Thiothixene & Arpiprazol & Prometazina & Diatrizoate \\
Loxapine & Zotepina & & Litio \\
Bromperidol & Amisulprida & & Phenelzine \\
Promazine & & & & Dosulepin \\
Clopentixol & & & Trimipramina \\
\hline
\end{tabular}

En todo paciente que ingresa a la Unidad Critica de Emergencia como Shock Trauma se da prioridad al riesgo de muerte y soporte de vida de la persona ${ }^{(6)}$, y es tratado como tal. Muchas entidades clínicas se manejan secundariamente e incluso son diagnosticadas posteriormente. Es lo que ocurre con el caso clínico referido, que recibió la atención como un shock distributivo-séptico, y luego se llegó al diagnóstico definitivo.

El bloqueo de receptores de dopamina a nivel del sistema nervioso central ${ }^{(7)}$ se ha descrito como la génesis de este síndrome, respaldado en evidencia como la asociación con los medicamentos que bloquean los receptores dopaminérgicos en el sistema nervioso central ${ }^{\left({ }^{8}\right)}$. El diagnóstico de SNM es clínico y, de acuerdo con los criterios del Manual diagnóstico y estadístico de los trastornos mentales (DSM-IV-TR), la elevación de CK en plasma constituye un criterio menor y no es preciso su presencia para realizar el diagnóstico (Tabla 3) (9). Sin embargo, dada la alta prevalencia de elevación sérica de CK en las fases iniciales se considera un criterio importante para el diagnóstico precoz (está presente en más del $90 \%$ de los casos), para normalizarse, habitualmente, el día $12{ }^{(8)}$. 
Tabla 3. Criterios diagnósticos del SNM según Manual diagnóstico y estadístico de los trastornos mentales (DSM-IV-TR)

\section{Criterios diagnósticos de SNM}

A. Aparición de rigidez muscular intensa y fiebre elevada asociada con la toma de medicación antipsicótica

B. Dos (o más) de los siguientes síntomas:

1. Diaforesis

2. Disfagia

3. Temblor

4. Incontinencia

5. Cambios en la conciencia que van desde confusión hasta coma

6. Mutismo

7. Taquicardia

8. Tensión arterial elevada o fluctuante

9. Leucocitosis

10. Hallazgos analíticos que indican lesión muscular (por ejemplo elevación de la concentración sérica de creatina cinasa).

C. Los síntomas de criterios $A$ y $B$ no se deben a una sustancia o a una enfermedad médica.

D. Los síntomas de criterios $A$ y $B$ no se explican mejor por la presencia de un trastorno mental.

El SNM es una urgencia neurológica, con un alto porcentaje de mortalidad y morbilidad. Así, el caso reportado por Vera et al., en la ciudad de Asunción (Paraguay) es similar al nuestro. Se trata de un paciente que ingresó al Servicio de Urgencias en mal estado general, febril, disneico, hipotenso, por lo que es ingresado a la Unidad de Reanimación donde es intubado y conectado a asistencia respiratoria mecánica; se realiza los exámenes de laboratorio de rutina, policultivos, antibioticoterapia empírica con diagnóstico probable de sepsis, punto de partida pulmonar. Posteriormente, la paciente persiste con rigidez muscular, le realizan una tomografía cerebral en la que no se evidencia imágenes sugestivas de patología cerebral; al ampliar anamnesis se reporta suspensión brusca de levodopa-carbidopa y es diagnosticado y tratado como SNM (10).

Otro caso similar al reportado por Vázquez Estévez et al. fue el de un paciente que presentó evento psicótico y se le administró olanzapina $20 \mathrm{mg} /$ día y risperidona 6 $\mathrm{mg} /$ día, a los 15 días presenta rigidez y alteración del sensorio es tratado como una sepsis foco a determinar posteriormente diagnóstico fue SNM. El paciente presentó evolución favorable a pesar del tiempo transcurrido ${ }^{(11)}$.

Remuñán Boue reporta un caso de paciente psiquiátrico que recibe haloperidol $5 \mathrm{mg}$ intramusculary trihexafenidilo (Parkisonil) de $2 \mathrm{mg}, 1$ tableta cada 6 horas. El paciente presenta alteración del estado de conciencia e hipertonía marcada, con tomografía cerebral y punción lumbar negativa, además presenta secreciones y se diagnostica como sepsis foco respiratorio. Pasa a cuidados intensivos con ventilación mecánica, se sospecha de SNM con CPK elevada, se le retira neurolépticos, se administra hidratación enérgica, dantroleno y bromocriptina; paciente con buena evolución es dado de alta ${ }^{(12)}$.

El SNM es poco frecuente, pero cuando se presenta tiene características de gran similitud al caso presentado; no es identificado al ingreso de urgencias y al persistir la sintomatología después de hacer el descarte de varias patologías se llega al diagnóstico final; como los casos descritos anteriormente.

Las complicaciones propias de esta enfermedad son múltiples, dentro de ellas, la depleción de volumen, secundaria a ella, el daño renal, y todos los cambios del medio interno, y el desequilibrio de la homeostasis. Además, el trastorno de sensorio que podría tener secuelas si no se realiza una neuroprotección adecuada. También debe considerarse las comorbilidades en los pacientes porque aumentan la morbimortalidad. Por último, el tratamiento de soporte es la piedra angular de la terapia médica, el pronóstico es bueno cuando se realiza un diagnóstico temprano y oportuno ${ }^{(13)}$.

El síndrome neuroléptico maligno es un cuadro muy raro, según múltiples fuentes; sin embargo, se debe tener en 
cuenta en los pacientes que, por algún motivo, tienen prescripción médica de antipsicóticos, trastorno de sensorio y rigidez muscular; siempre y cuando se hayan descartado otras causas estructurales del sistema nervioso central (SNC) como accidente cerebro-vascular (ACV) isquémico o hemorrágico, y problemas sépticos como meningitis. El manejo de fondo es la terapia de soporte con fluidoterapia plena, soporte ventilatorio, y corrección de los desórdenes metabólicos. Existe medicación para este cuadro como dantroleno y bromocriptina, pero su uso solo será beneficioso en los primeros días, y como terapia complementaria y no terapia principal.

Contribuciones de los autores: FRC ha participado en la concepción, diseño del artículo y aprobación de la versión final del manuscrito. VRC elaboró el primer borrador del artículo. VRC y FRC han realizado la revisión crítica del contenido.

\section{REFERENCIAS BIBLIOGRÁFICAS}

1. León-Caballero J, Alba-Pale L, Salgado-Serrano P, Pérez-Solá V. Neuroleptic malignant syndrome with slight elevation of creatine kinase in serum: a brief review. Acta Esp Psiquiatr. 2015 Sep; 43(5): 194-6.

2. Gagnier JJ, Kienle G, Altman DG, Moher D, Sox H, Riley D, et al. The CARE Guidelines: Consensus-based Clinical Case Reporting Guideline Development. Glob Adv Health Med. 2013 Sep; 2(5): 38-43.

3. Fernández $M$, Lago $L$, Alonso MG, Guede A, Benavente JL, Olivares JM. Serotonin syndrome versus neuroleptic malignant syndrome: a case report. Acta Esp Psiquiatr. 2018 Mar; 46(2): 68-74.

4. González Muñoz A, Pérez Sánchez JR, Contreras Chicote A. Diagnóstico diferencial etiopatogénico de la demencia: otras demencias. Medicine - Programa de Formación Médica Continuada Acreditado. 2015 Feb; 11(72): 4345-9.

5. Ninčević Ž, Lasić D, Glavina T, Mikačić M, Carev M, Podrug K. Quetiapine poisoning associated with neuroleptic malignant syndrome, rhabdomyolysis and renal failure: a case report. Psychiatr Danub. 2017 Mar; 29(1): 84-6.

6. Singer B, Reynolds JC, Lockey DJ, O’Brien B. Pre-hospital extra-corporeal cardiopulmonary resuscitation. Scand J Trauma Resusc Emerg Med. 2018; 26(1): 21.

7. Wachtel L, Commins E, Park M, Rolider N, Stephens R, Reti I. Neuroleptic malignant syndrome and delirious mania as malignant catatonia in autism: prompt relief with electroconvulsive therapy. Acta Psychiatr Scand. 2015 Oct; 132(4): 319-20.

8. Loeb E, Madigand J, Alexandre J, Dollfus S, Coquerel A, Fedrizzi S. Neuroleptic malignant syndrome and catatonia overlapping: 2 case reports. Psychopharmacology (Berl). 2015 Jul; 232(14): 2643-4.

9. Lang FU, Lang S, Becker T, Jäger M. Neuroleptic malignant syndrome or catatonia? Trying to solve the catatonic dilemma. Psychopharmacology (Berl). 2015 Ene; 232(1): 1-5.

10. Vera R, Denis A, Recalde A, Paats A, Esteche A. Neuroleptic malignant-like síndrome. Rev Virtual Soc Parag Med Int. 2017 Sep; 4(2): 49-53.

11. Vázquez Estévez R, Morillo Blanco ML, Molina Castillo JJ, Rubio Merino MI, Arenas García JA. Síndrome neuroléptico maligno: dificultades en el diagnóstico diferencial. Ver Psiquiatr Salud Ment. 2016 Sep; 23(3): 122-4.

12. Remuñán Boue $C$, Rondón J, Davas Santana R, Vázquez Vázquez L. Sindrome neuroléptico maligno. Rev Cub Med Int Emerg. 2004; 3(1): 46-51

13. Chackupurakal R, Wild U, Kamm M, Wappler F, Reske D, Sakka SG. Neuroleptic malignant syndrome: rare cause of fever of unknown origin. Anaesthesist. $2015 \mathrm{Jul}$; 64(7): 527-31.

Fuentes de financiamiento

Este artículo ha sido financiado por los autores.

\section{Conflictos de interés}

Al momento de la concepción de la publicación del artículo, los autores laboraron en EsSalud.

\section{Correspondencia:}

Fanny Elizabeth Ramírez Calderón

Dirección: Domingo Cueto N120, Jesús María. Lima, Perú.

Teléfono: 945460084

Correo electrónico: racaelifa@hotmail.com

\section{Recibido: 20 de noviembre de 2018. \\ Evaluado: 04 de abril de 2019. \\ Aprobado: 13 de abril de 2019.}

(c) La revista. Publicado por Universidad de San Martín de Porres, Perú. (c) $)_{\text {Br }}$ Licencia de Creative Commons Artículo en acceso abierto bajo términos de Licencia Creative Commons Atribución 4.0 Internacional. (http://creativecommons.org/licenses/by/4.0/)

\section{ORCID iDs}

Fanny Elizabeth Ramírez Calderón (D) https://orcid.org/0000-0003-3992-983X Víctor Ramos Cabrera $\quad$ (D) https://orcid.org/0000-0001-5942-1086 